\title{
The NA62 Experiment at CERN: Status of the Construction
}

\author{
Ferdinand Hahn ${ }^{1}$ for the NA62 Collaboration ${ }^{2}$ \\ CERN \\ 1211 Geneva 23, Switzerland \\ E-mail: Ferdinand.Hahn@cern.ch
}

The NA62 collaboration is building a new experiment tailored to measure the ultra-rare kaon decay $K^{+} \rightarrow \pi^{+} v \bar{v}$. The first physics run is planned in autumn 2014. This paper presents the detectors of the new experiment and reports on the status of construction as well as first results from the Technical Run in November 2012.

2013 Kaon Physics International Conference

April 29-May 1, 2013

University of Michigan, Ann Arbor, Michigan - USA

\footnotetext{
${ }^{1}$ Speaker

2 The NA62 Collaboration: G. Aglieri Rinella, F. Ambrosino, B. Angelucci, A. Antonelli, G. Anzivino, R. Arcidiacono, I. Azhinenko, S. Balev, J. Bendotti, A. Biagioni, C. Biino, A. Bizzeti, T. Blazek, A. Blik, B. BlochDevaux, V. Bolotov, V. Bonaiuto, D. Britton, G. Britvich, N. Brook, F. Bucci, V. Buescher, F. Butin, E. Capitolo, C. Capoccia, T. Capussela, V. Carassiti, N. Cartiglia, A. Cassese, A. Catinaccio, A. Cecchetti, A. Ceccucci, P. Cenci, V. Cerny, C. Cerri, A. Cecchetti, O. Chikilev, R. Ciaranfi, G. Collazuol, P. Cooke, P. Cooper, G. Corradi, E. Cortina Gil, F. Costantini, A. Cotta Ramusino, D. Coward, G. D'Agostini, J. Dainton, P. Dalpiaz, H. Danielsson, J. Degrange, N. De Simone, D. Di Filippo, L. DiLella, N. Dixon, N. Doble, V. Duk, V. Elsha, J. Engelfried, V. Falaleev, R. Fantechi, L. Federici, M. Fiorini, J. Fry, A. Fucci, S. Gallorini, L. Gatignon, A. Gianoli, S. Giudici, L. Glonti, A. Goncalves Martins De Oliveira, F. Gonnella, E. Goudzovski, R. Guida, E. Gushchin, F. Hahn, B. Hallgren, H. Heath,F. Herman, E. Iacopini, O. Jamet, P. Jarron, K. Kampf, J. Kaplon, V. Karjavin, V. Kekelidze, A. Khudyakov, Yu. Kiryushin, K. Kleinknecht, A. Kluge, M. Koval, V. Kozhuharov, M. Krivda, J. Kunze, G. Lamanna, C. Lazzeroni, R. Leitner, R. Lenci, M. Lenti, E. Leonardi, P. Lichard, R. Lietava, L. Litov, D. Lomidze, A. Lonardo, N. Lurkin, D. Madigozhin, G. Maire, A. Makarov, I. Mannelli, G. Mannocchi, A. Mapelli, F. Marchetto, P. Massarotti, K. Massri, P. Matak, G. Mazza, E. Menichetti, M. Mirra, M. Misheva, N. Molokanova, J. Morant, M. Morel, M. Moulson, S. Movchan, D. Munday, M. Napolitano, F. Newson, A. Norton, M. Noy, G. Nuessle, V. Obraztsov, S. Padolski, R. Page, T. Pak, V. Palladino, A. Pardons, E. Pedreschi, M. Pepe, F. Perez Gomez, F. Petrucci, R. Piandani, M. Piccini, J. Pinzino, M. Pivanti, I. Polenkevich, I. Popov, Yu. Potrebenikov, D. Protopopescu, F. Raffaelli, M. Raggi, P. Riedler, A. Romano, P. Rubin, G. Ruggiero, V. Russo, V. Ryjov, A. Salamon, G. Salina, V. Samsonov, E. Santovetti, G. Saracino, F. Sargeni, S. Schifano, V. Semenov, A. Sergi, M. Serra, S. Shkarovskiy, A. Sotnikov, V. Sougonyaev, M. Sozzi, T. Spadaro, F. Spinella, R. Staley, M. Statera, P. Sutcliffe, N. Szilasi, D. Tagnani, M. Valdata-Nappi, P. Valente, V. Vassilieva, B. Velghe, M. Veltri, S. Venditti, M. Vormstein, H. Wahl, R. Wanke, P. Wertelaers, A. Winhart, R. Winston, B. Wrona, O. Yushchenko, M. Zamkovsky, A. Zinchenko.
} 


\section{Introduction}

NA62 is the successor of a previous kaon decay experiment (NA48) operated in the same experimental area at the CERN SPS. NA62 re-uses large parts of the existing infrastructure, and it performed its first data talking in 2007 with the detectors available from NA48. In December 2008 the CERN Research Board has authorized the collaboration to build new detectors which are tailored to measure the very rare decay $K^{+} \rightarrow \pi^{+} v \bar{\nu} \quad\left(\mathrm{SM} \mathrm{BR}=(8.5 \pm 0.7) * 10^{-11}[1]\right)$.

The timeline of the experiment foresees 5 years (2009-2014) of detector construction interleaved by a first run in 2012 and physics data talking starting in autumn 2014.

The new experiment uses high energy protons from the SPS (400 GeV/c) to produce a secondary hadron beam (6\% kaons) of $75 \mathrm{GeV} / \mathrm{c}$ and high intensity $(750 \mathrm{MHz}$ ).

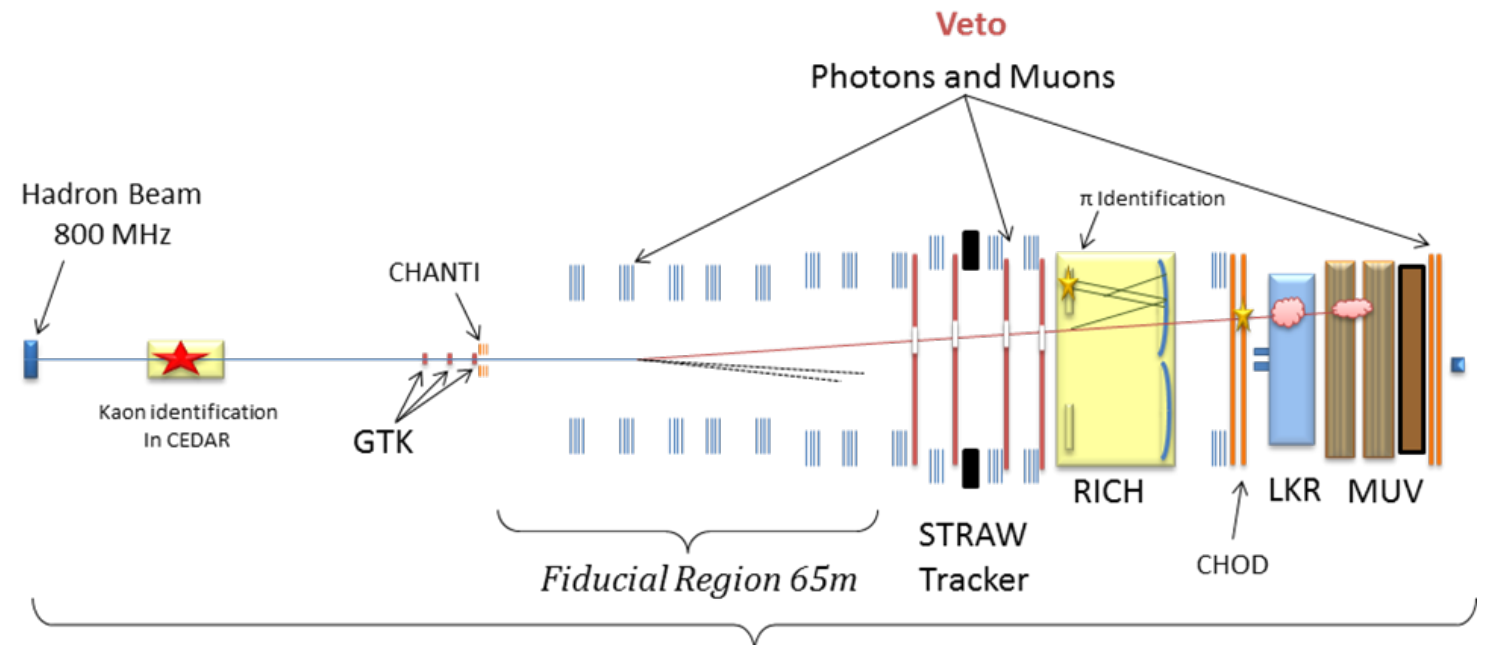

Total Length $270 \mathrm{~m}$

Figure 1 Schematic view of the NA62 Experiment.

\section{Detector Description}

The experimental set-up extends from the beryllium target to the beam dump over about $270 \mathrm{~m}$. The first $100 \mathrm{~m}$ are covered by beam elements and two detectors measuring the incoming beam (KTAG and GTK). Downstream of the last GTK station starts a $65 \mathrm{~m}$ long fiducial region in which useful $\mathrm{K}^{+}$decays are registered. The main detectors measuring the decay products start from there and range over a $170 \mathrm{~m}$ long region ending shortly before the beam dump (see Figure 1).

The main ingredients for detecting the very rare $K^{+} \rightarrow \pi^{+} v \bar{v}$ decays are the following:

- High beam intensity and fast timing: The intense beam rate $(750 \mathrm{MHz})$ and the need to match the incoming $\mathrm{K}^{+}$with the outgoing $\pi^{+}$defines the timing requirements of the experiment. Without precise timing, an accidental beam 
particle can be mistaken for the decaying kaon and be associated to the $\pi^{+}$. With 150 ps time resolution for the beam particles and 100 ps for the $\pi^{+}$tracks, the fraction of events with a wrong association can be kept below $1 \%$ once appropriate spatial cuts (e.g. cuts based on the closest distance of approach between the pion and kaon track candidates) are applied.

- Ultra-light tracking detectors: The most discriminating variable to reject the high branching ratio kaon decays $\left(\mathrm{K}^{+} \rightarrow \pi^{+} \pi^{0}, \mathrm{~K}^{+} \rightarrow \mu^{+} v\right.$ and $\left.\mathrm{K}^{+} \rightarrow \pi^{+} \pi^{-} \pi^{0}\right)$ from signal is the squared missing mass ${ }^{3}$. Backgrounds from these decays can, however, enter the signal regions because of kinematic resolution effects caused, for instance, by multiple scattering. It is therefore important to keep the spectrometer material budget as low as possible.

- Hermetic vetoing for photons and muons: the kinematic suppression of $\mathrm{K}^{+} \rightarrow$ $\pi^{+} \pi^{0}$ and $\mathrm{K}^{+} \rightarrow \mu^{+} v$ must be complemented by efficient direct vetoing of photons and muons. The photon vetos cover polar angles from 0 to 50 mrad, they are optimized for good detection efficiency of high energy photons. The primary muon rejection is done in the trigger (L0) by the fast muon veto system (MUV3).

- Particle ID: the muon rejection is enhanced by a $17 \mathrm{~m}$ long RICH detector separating pions from muons in a momentum range between 15 and 35 GEV/c. The positive identification of the $\mathrm{K}^{+}$in the beam is done by the KTAG. This is important because $93 \%$ of the beam particles are $\pi^{+}$or protons. By flagging the the kaons in the beam one can reject effectively beam gas events from other beam particles, which could mimic the signature of the signal events.

\subsection{The K12 Beam}

NA62 aims to collect about $10^{13} \mathrm{~K}^{+}$decays requiring high beam intensities. The new beam line installed at the CERN SPS extraction line provides a secondary hadron beam of $75 \mathrm{GeV} / \mathrm{c}$ central momentum. The characteristics of the slowly extracted beam is a flat intensity over 6s (3s in 2014) spill duration with a cycle time 40s (20s in 2014). During the spill the beam has a flux rate of $750 \mathrm{MHz}$ (see Table 1).

Table 1 K12 Beam intensities and number of kaon decays.

\begin{tabular}{|l|c|}
\hline $400 \mathrm{GeV} / \mathrm{c}$ Protons on Target / s & $1.1 \times 10^{12}$ \\
\hline $75 \mathrm{GeV} / \mathrm{c}$ Hadrons / s (6\% kaons) & $750 \times 10^{6}$ \\
\hline $75 \mathrm{GeV} / \mathrm{c} \mathrm{K}^{+}$decays / s & $4.5 \times 10^{6}$ \\
\hline Expected $\mathrm{K}^{+}$decays /y & $\approx 5 \times 10^{12}$ \\
\hline
\end{tabular}

\subsection{The KTAG}

The KTAG identifies the $\mathrm{K}^{+}$component in the beam with respect to the other beam particles by employing an upgraded differential Cherenkov (CEDAR) counter from the SPS at CERN. The essential feature of this detector is a precise timing $\left(\sigma_{t}=100 \mathrm{ps}\right)$ in

${ }^{3} \mathrm{~m}_{\text {miss }}^{2} \equiv\left(\mathrm{P}_{\mathrm{K}}-\mathrm{P}_{\pi}\right)^{2} \quad \mathrm{P}_{\mathrm{K}}$ denotes the 4-momentum of the parent particle assumed to be a kaon and $\mathrm{P}_{\pi}$ is the 4momentum of the decay particle assumed to be a $\pi^{+}$. 
order to reduce accidental overlaps with beam gas events. The original CEDAR detector consists of a $4.35 \mathrm{~m}$ long radiator vessel filled with gas of a precisely controlled pressure. At the end of the vessel Cherenkov light is reflected by a mangin mirror onto a circular diaphragm with adjustable aperture width. The light exists the vessel through eight quartz windows at the upstream end; in the original version of the detector eight photomultipliers were mounted in this position to detect the Cherenkov light (Figure 2 left)[2].
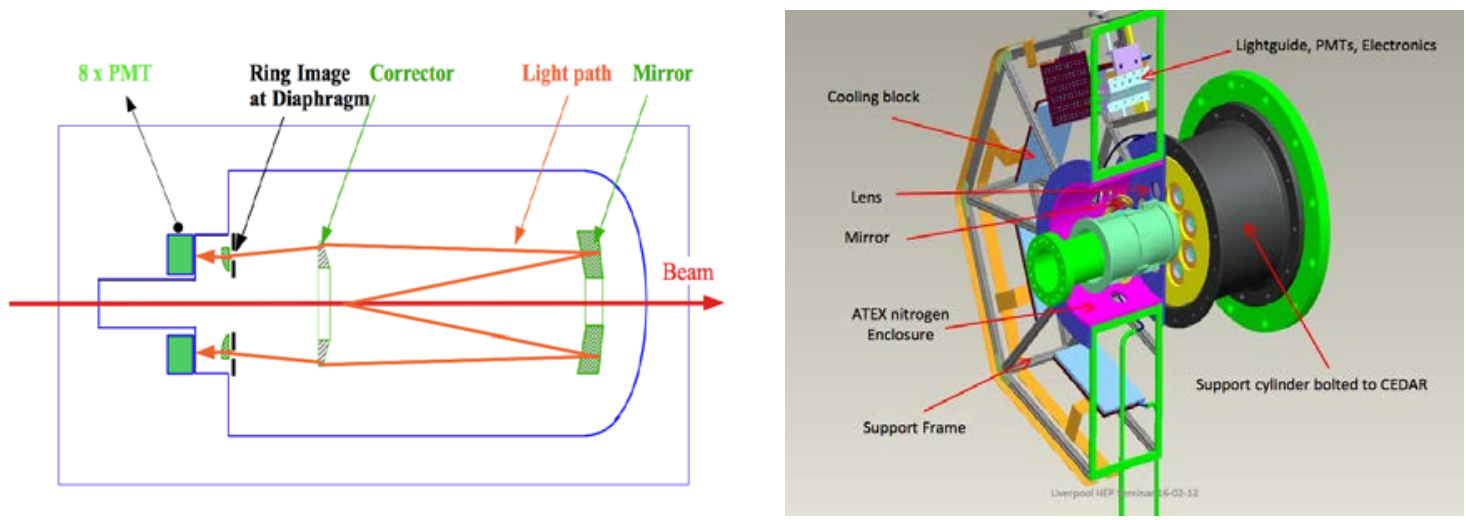

Figure 2 Schematic drawing of the original CEDAR (left). The new optics and electronics of the KTAG (right).

The kaon rate in the hadron beam of NA62 is $50 \mathrm{MHz}$, which is far too high for a standard CEDAR. The KTAG has therefore an additional external optic projecting the eight light spots radially out, so that the light is distributed over 48 photomultipliers ${ }^{4}$ in each spot (see Figure 2 right).

The crucial elements of the new KTAG are the photo detectors, the front-end electronics and the readout system providing high efficiency, excellent time resolution (100 ps) and high rate capability $(50 \mathrm{MHz})$.

- Photo-detectors: PMT R7400/U-03 and R9880/U-03 from Hammatsu

- Front-electronics: uses the NINO Board (64 channels per board) with ultra-fast amplifier and discriminator [3], the input is a differential signal from the PMT.

- The read-out is performed by the TEL62 board, which provides the trigger and the DAQ, hosting 4 daughter HPTDC boards (128 channels each).

\subsection{The GTK}

The beam spectrometer, called Gigatracker (GTK), consists of three Si micro-pixel detectors matching the beam dimensions. These stations are placed in vacuum and are crossed by the full beam intensity of about $750 \mathrm{MHz}$ (see Figure 3). The GTK provides a time resolution of better than $150 \mathrm{ps,} \mathrm{which} \mathrm{is} \mathrm{vital} \mathrm{to} \mathrm{associate} \mathrm{the} \mathrm{right} \mathrm{beam} \mathrm{particle}$ to the decay vertex. Four dipole magnets provide an achromatic (no net bending) spectrometer for particles of any momentum (Figure 4). The momentum resolution is

\footnotetext{
${ }^{4}$ In the final phase the number of photo detectors per light spot will be increased to 64 per light spot.
} 
expected to be $0.2 \%$ RMS and the angular resolution $15 \mu \mathrm{rad}$ on an event-by-event basis.

A critical requirement is a material budget of $0.5 \%$ of $\mathrm{X} 0$, all components are therefore optimized in that respect. The GTK is a hybrid detector with a sensor (thickness of 200 $\mu \mathrm{m})$ flip-chip bonded to 10 read-out chips (thickness $100 \mu \mathrm{m}$ ). On the backside of the read-out chips the assembly is glued to a micro-channel cooling plate to cool down the chips to -5 degree $\mathrm{C}$. The total thickness of one station is $<500 \mu \mathrm{m}$.

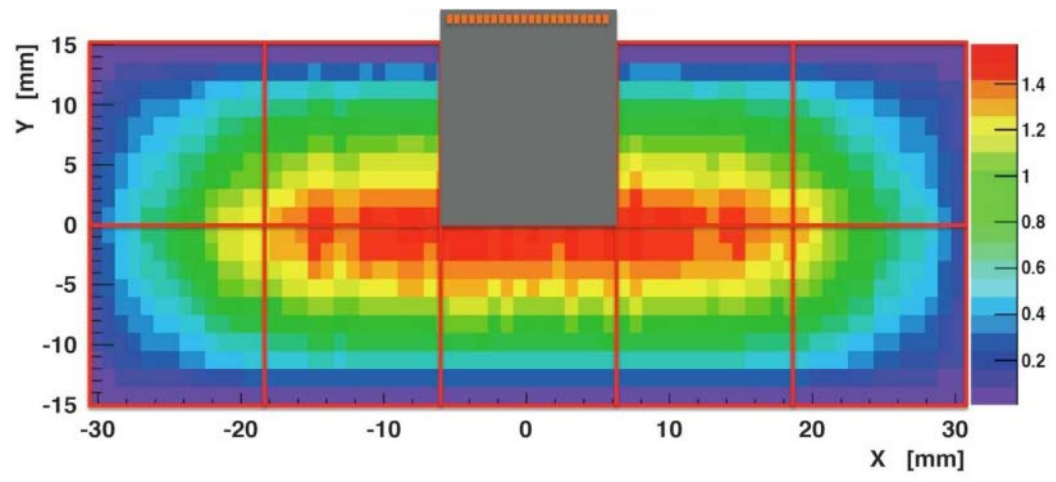

Figure 3 Beam intensity distribution in GTK3 in $\mathrm{MHz} / \mathrm{mm}^{2}$. The active area of the sensor $(27.0 \times 60.8$ $\mathrm{mm}^{2}$ ) is bump bonded to 10 readout chips (one is schematically shown).

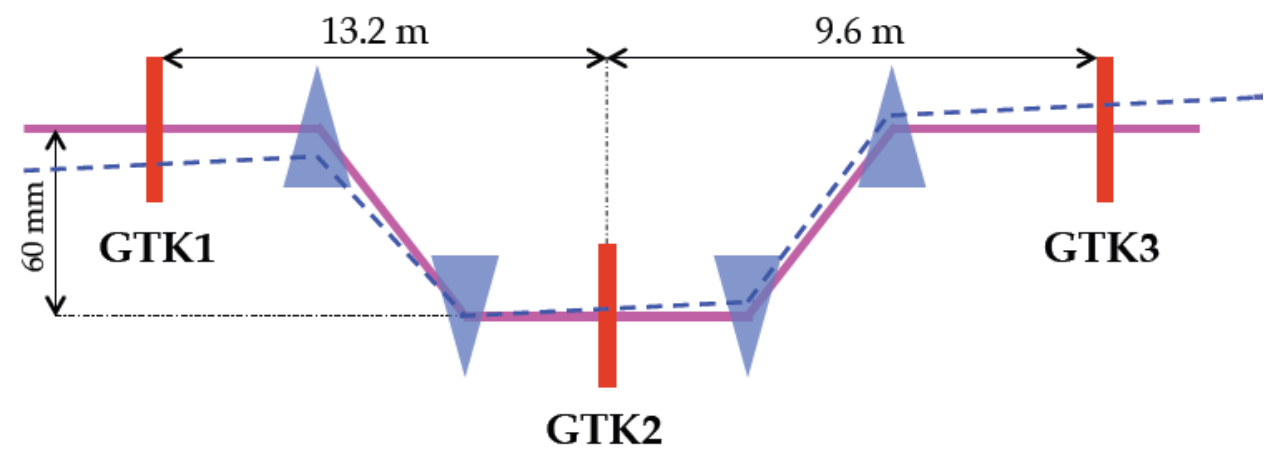

Figure 4 The GTK stations are mounted in the beam line between 4 achromat magnets measuring time, direction and momentum of all beam particles.

\subsection{The CHANTI}

The last GTK station is complemented by 6 stations of 'guard-ring' counters called CHANTI to reduce critical background from inelastic interactions from beam particles in the last GTK station. Each guard-ring station consist of 4 layers of triangular shaped scintillator bars (Figure 5) readout by wave-length-shifting fibers and Silicon Photomultipliers. 

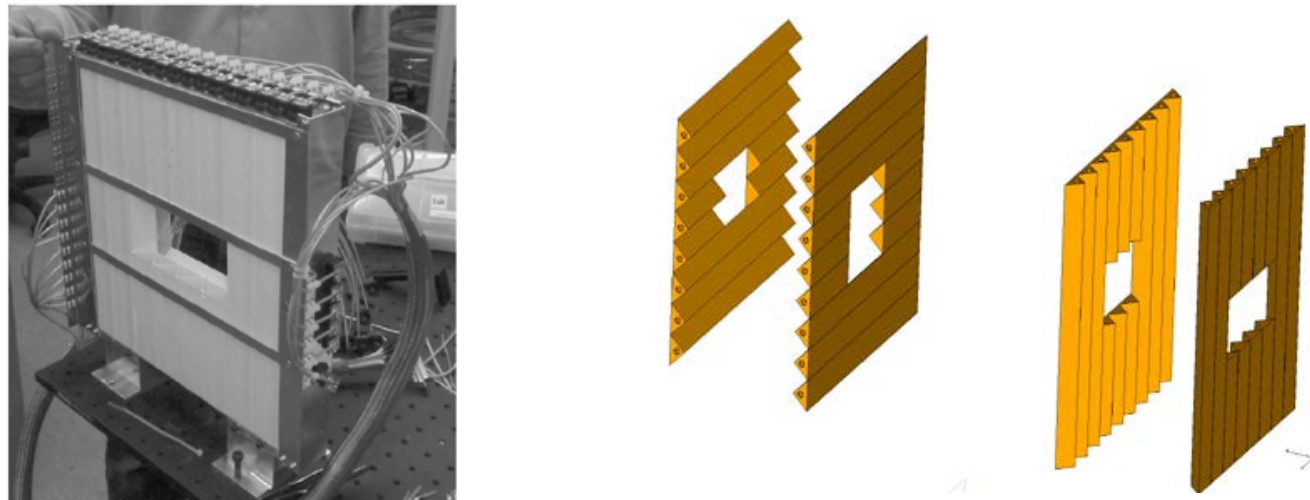

Figure 5 One CHANTI station made of 4 layers of scintilators (triangular shape) with WLS fiber readout.

\subsection{The Photon Vetos}

The suppression of possible background from $\mathrm{K}^{+} \rightarrow \pi^{+} \pi^{0}$ decays determines the design of the photon veto system. One requires an overall inefficiency of about $10^{-8}$ for the $\pi^{0}$ detection.

In the offline-analysis, the $\pi^{+}$momentum will be required to be less than $35 \mathrm{GeV} / \mathrm{c}$. In this way, the momentum of the $\pi^{0}$ amounts to at least $40 \mathrm{GeV} / \mathrm{c}$. As a consequence the NA62 design needs very high detection efficiency for high energy photons, the inefficiencies have to be below $10^{-5}$ for photons above $10 \mathrm{GeV}$, and less than $10^{-3}$ for photons above $1 \mathrm{GeV}$. The veto system covers an acceptance from 0 to $50 \mathrm{mrad}$ from the $\mathrm{K}^{+}$decay vertex with respect to the beam line.

\subsubsection{Large Angle Photon Veto (LAV)}

The Large Angle Veto (LAV) detector is one part of the overall photon veto system in the NA62 experiment. It consists of 12 stations distributed along the decay volume and spaced by $6 \mathrm{~m}$ in the upstream region (see Figure 6) and by up to $20 \mathrm{~m}$ for the most downstream station. Eleven (out of 12) LAV detectors are installed inside the vacuum tank, they cover the angular region between $8.5 \mathrm{mr}$ and 50 mrad.
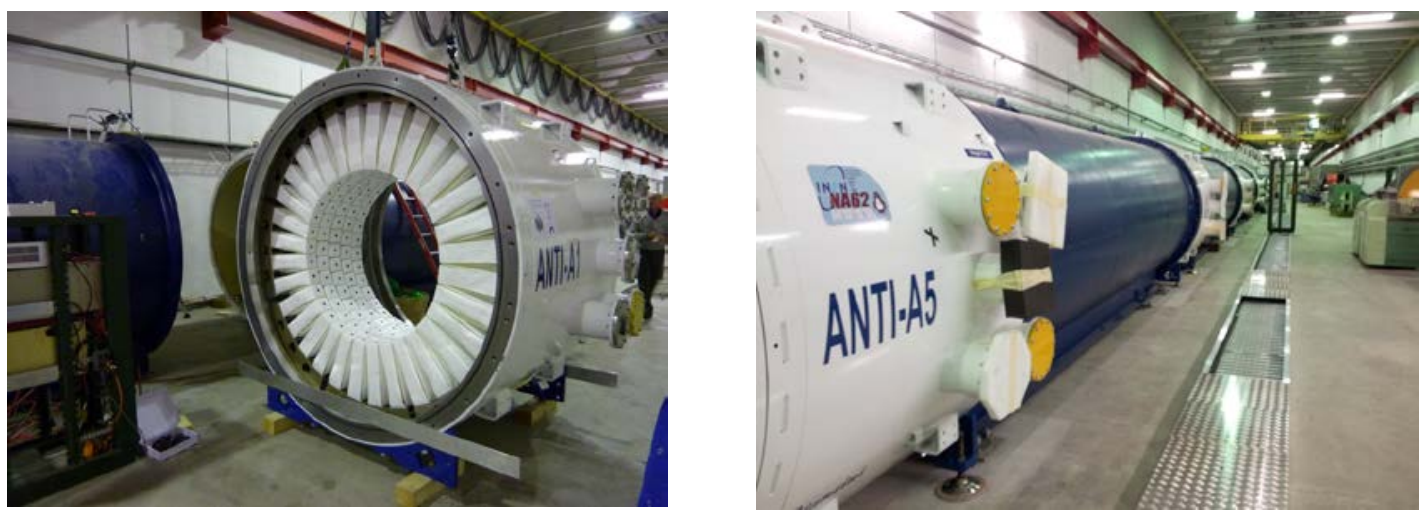

Figure 6 Photos from the installation of the LAV detector

The basic building blocks of this detector are lead glass crystals with attached photomultipliers from the former OPAL electromagnetic calorimeter. Each LAV 
stations is made up of 4 or 5 rings of crystals, which are slightly staggered in azimuth providing a complete hermeticity for tracks in this angular region

\subsubsection{Small Angle Photon Veto (IRC and SAC)}

Two small shaslik calorimeters

As shown in Figure 7 , two Small Angle calorimeters are employed to veto photons at very small angles:

1. The IRC covering the region around the inner radius of the Liquid Krypton Calorimeter.

2. The SAC situated behind the experimental cavern in the prolongation of the nondeviated axis covering the angular region down to 0 degrees.

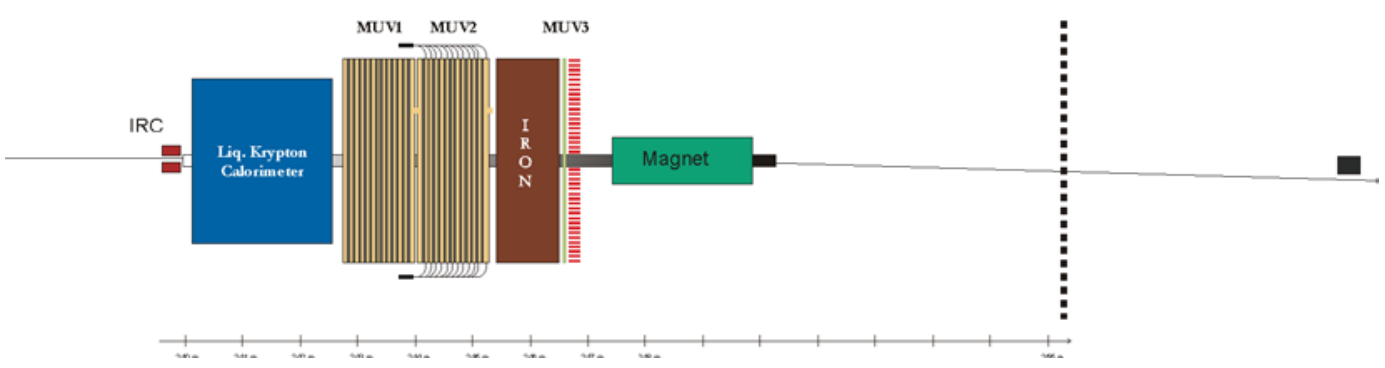

Figure 7 The IRC (infront of the LKR) and the SAC (at the end of the beam line) are used to veto photons at very small angle. The hadron calorimeters MUV1 and MUV2 are located directly after the LKR, the fast Muon Veto MUV3 follows behind $80 \mathrm{~cm}$ of iron.

Both detectors are small calorimeters made by alternating layers of lead and scintillators with fiber readout (shashlik). The fibers are readout by photomultipliers with ADC or TDC readout.

\subsubsection{The Liquid Krypton Calorimeter (LKR)}

NA62 re-uses the Liquid Krypton Calorimeter from NA48 as a veto for photons in the 1-10 mrad region. Measurements based on NA48 data have demonstrated the capability of the calorimeter to reach the required veto performance.

The LKR is a quasi-homogeneous calorimeter filled with about 9,000 liters of liquid Krypton, the active surface extends from the beam pipe $(\mathrm{R}=8 \mathrm{~cm})$ to a radius of $128 \mathrm{~cm}$, the thickness is $127 \mathrm{~cm}$ (27 radiation length). The sensitive area is divided in 13,248 longitudinal cells of about $2 \times 2 \mathrm{~cm}$ cross-section [4]. To satisfy the NA62 rate requirements the calorimeter will be equipped with new Calorimeter REAdout Modules (called CREAM). The CREAM readout (see Figure 8) provides FlashADC readout with a sampling rate of $40 \mathrm{MHz}$. The digitized inputs are stored in a circular memory with sufficient depth to buffer up to $10 \mathrm{~ms}$ of raw data. Upon receipt of the L0 trigger the digitizations of the selected timestamps are transferred into the L0 event buffer, which is able to accommodate a $1 \mathrm{MHz}$ average L0 rate during $10 \mathrm{~s}$ (spill length). After an L1 trigger, the events are available for readout via the Gigabit Ethernet link with an optional zero suppression processing. During the readout process, the CREAM is still able to store new data in the circular and L0 event buffers. The acquisition process is therefore "dead-time-less" as long as the L0 event buffer is not full [5]. In parallel to 
the normal read-out the CREAM will produce energy sums for groups of 16 cells, they are sent to the LKR L0 trigger system from which they provide energy information for the L0 trigger.

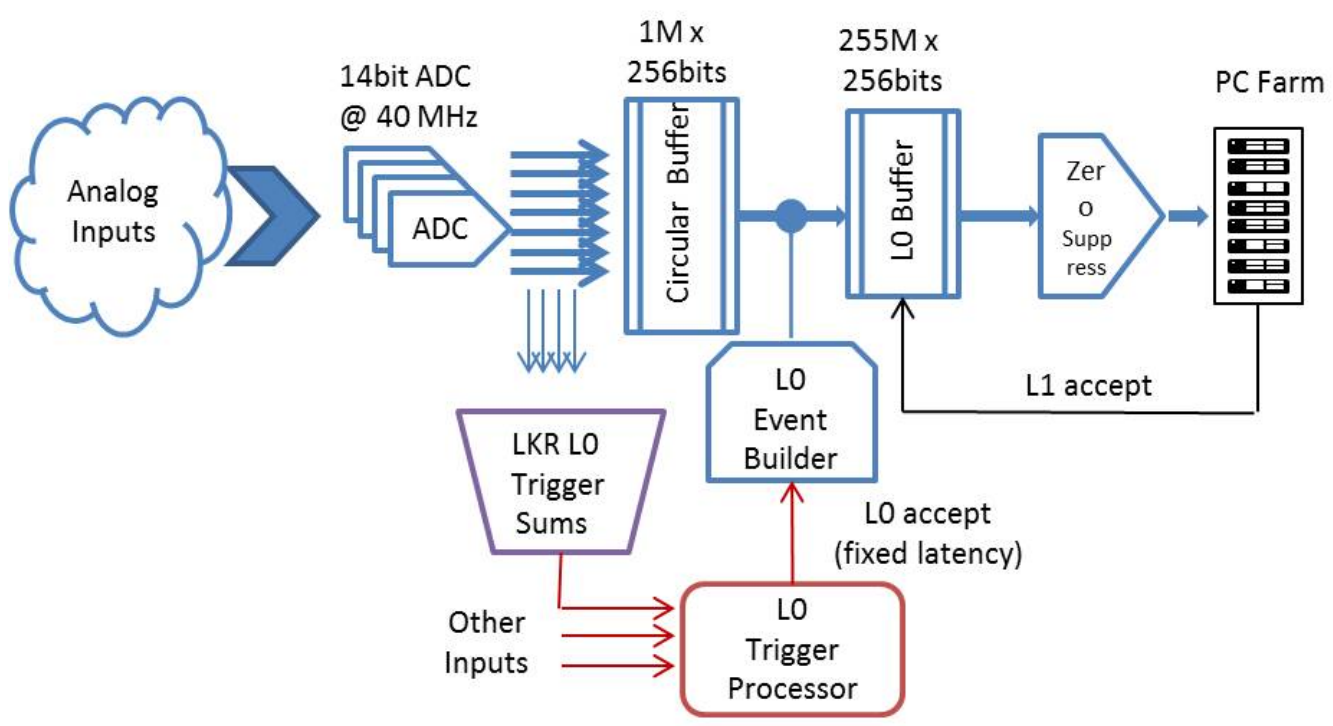

Figure 8 Schematic of the CREAM readout.

\subsection{Straw Tracker}

The STRAW Tracker measures the coordinates and momentum of secondary charged particles originating from the decay region. To minimize multiple scattering the chambers are built of ultra-light material and are installed inside the vacuum tank. The four Straw Chambers are intercepted in the middle by a large aperture dipole magnet (MNP33), providing a vertical B-field of 0.36T (see Figure 9). Each chamber comprises 1,792 straws, which are positioned in four "Views" providing a measurement of four coordinates (see Figure 10)[2].

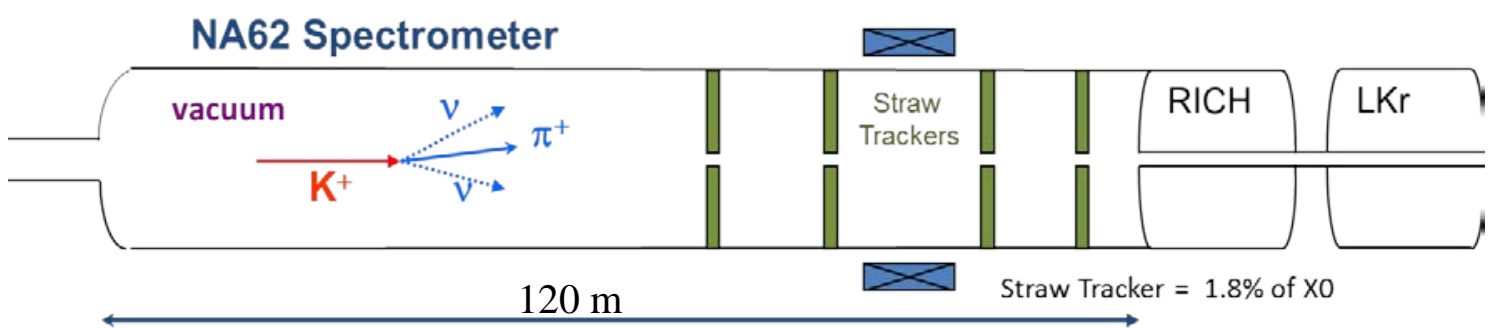

Figure 9 Schematic drawing of the NA62 spectrometer. Four Straw Chambers are installed inside the vacuum tank interleaved by a dipol magnet in the midlle. 

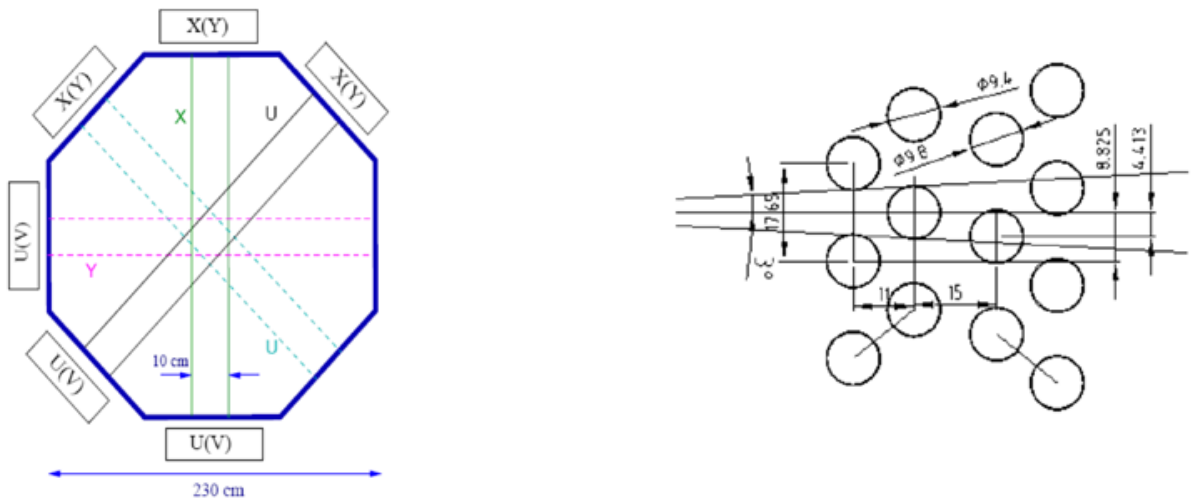

Figure 10 One Straw Chamber (left) measures 4 coordinates (called views). Near the middle of each view a few straws are left out forming a hole for the beam passage. Each view (right) includes four layers of straws providing typically three measured points per track.

The straws are manufactured from thin $(36 \mu \mathrm{m})$ polyethylene terephthalate (PET) foil that is longitudinally welded (ultra-sonic weld) to form a tube. Its inner surface has a metal coating $(\mathrm{Cu} / \mathrm{Au})$ to provide electrical conductance for the cathode. The straw length is $2.1 \mathrm{~m}$ and the diameter $9.8 \mathrm{~mm}$. One of the most important features of the straw is their leak tightness and their mechanical stability so that they can be installed inside the vacuum tank [2].

As shown Figure 11, half a straw chamber (2 Views) was successfully installed and operated during the Technical Run in autumn 2012.
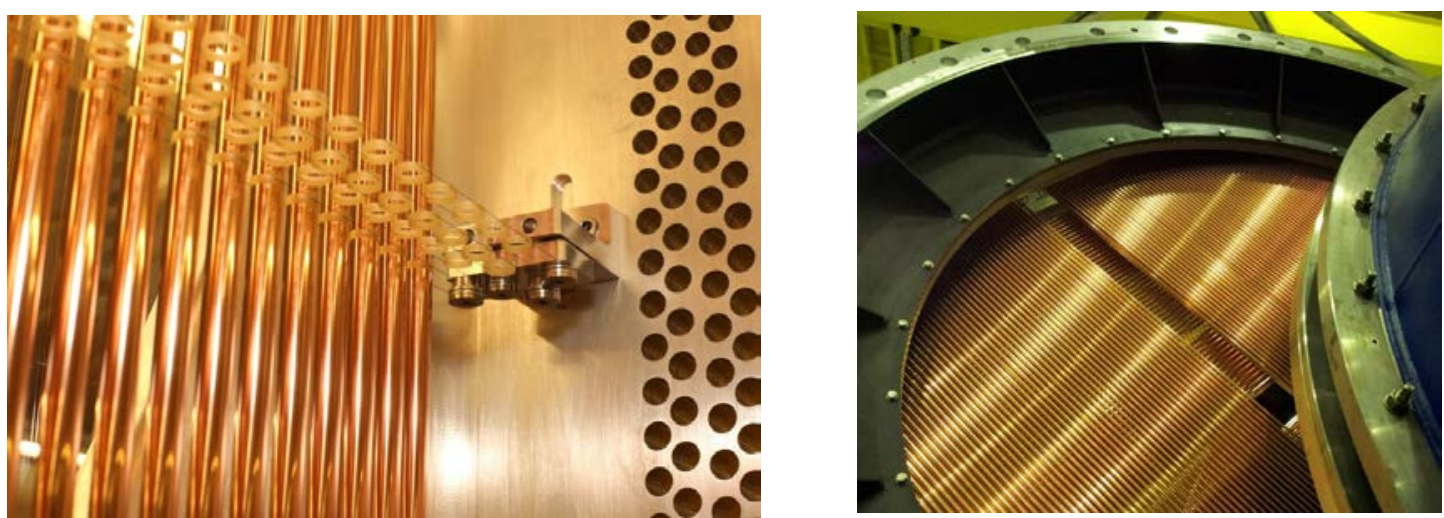

Figure 11 Picture from the straw chamber construction (left) and installation for the Technical Run (right).

\subsection{The RICH}

The RICH detector is required to improve the rejection of background from $\mathrm{K}^{+}->\mu^{+} \mathrm{v}$ decays providing positive identification of the Muon in the background events and an identification of the Pion in the signal events. 
The RICH Detector will:

- separate $\pi^{+}$from $\mu^{+}$between 15 and $35 \mathrm{GeV} / \mathrm{c}$ momentum providing a muon suppression factor of at least $10^{-2}$

- measure the pion crossing time with a resolution of about $100 \mathrm{ps}$;

- $\quad$ produce the L0 trigger for a charged track.

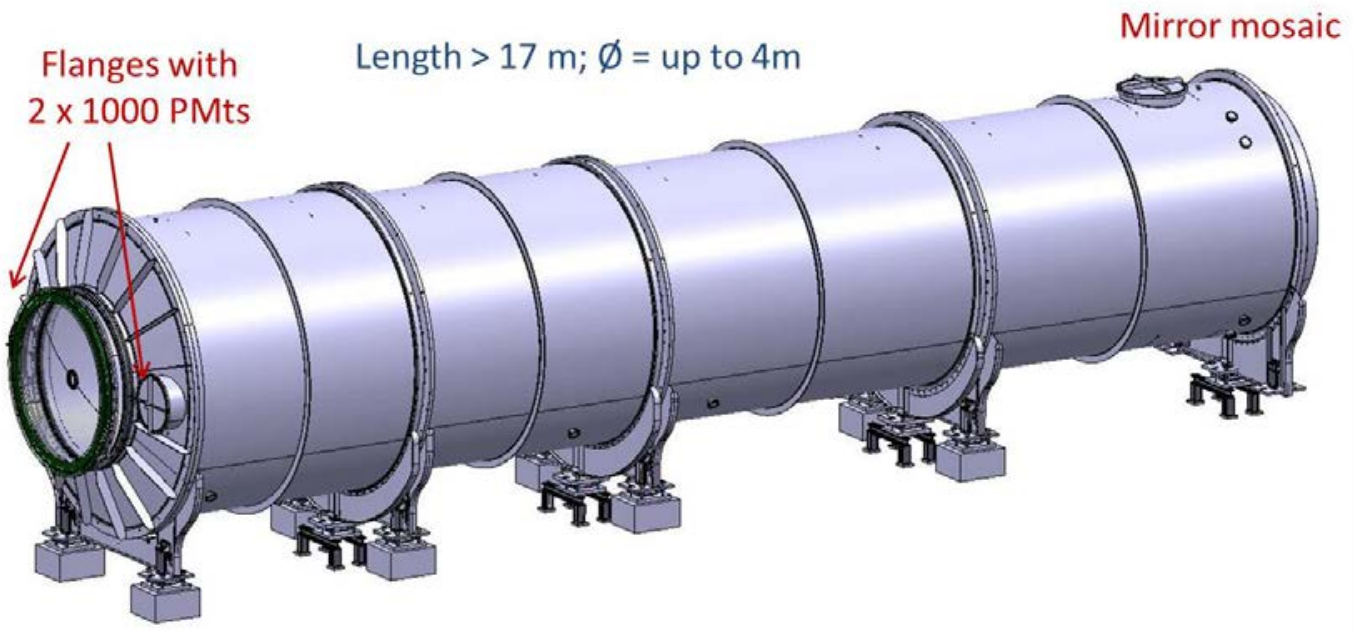

Figure 12 The Rich radiator vessel is a large vessel ( $>17 \mathrm{~m}$, up to $4 \mathrm{~m}$ in diameter). The downstream end contains a mosaic of sperical mirrors reflecting the Cherenkov light, onto two flanges equipped with 2,000 photomultipliers situated at the upstream end.
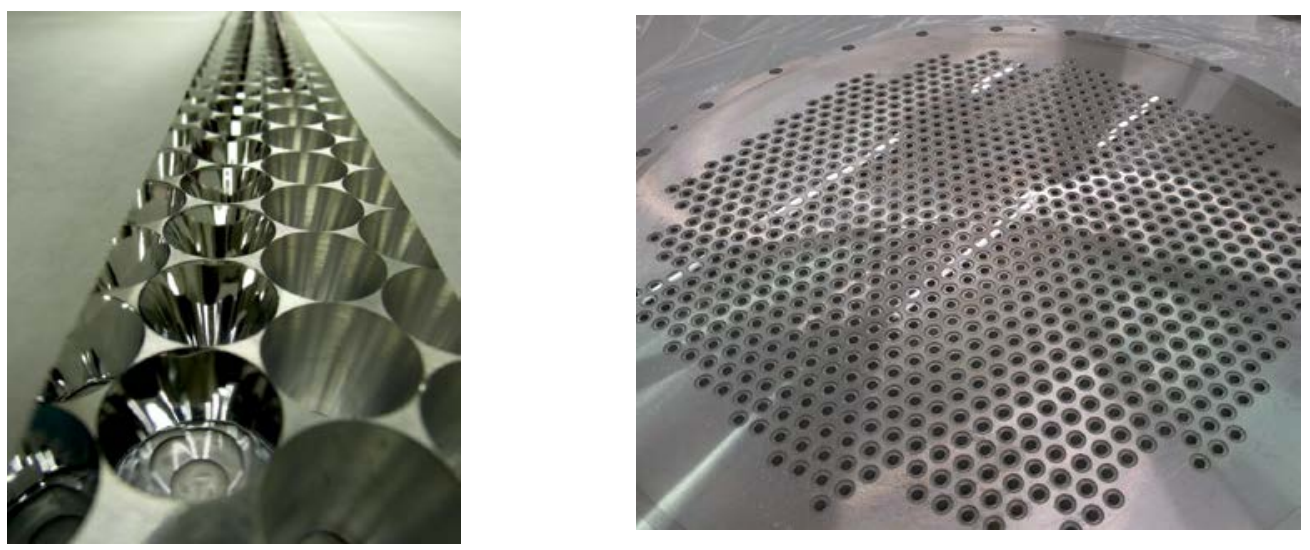

Figure 13 Construction of the quartz window flange. Left: On the inside of the flange are cones with aluminium coated myler to reflect the light onto the quartz window. Right: The flange from the outside showing showing 1,000 quartz windows.

The RICH is situated between the last Straw Station (210m from the target) and the last Large Angle Veto station. It consists of a cylindrical vessel (Length $\sim 18 \mathrm{~m}, \varnothing=3.2-$ $4.0 \mathrm{~m}$ ) with the beam pipe passing in its center. The vessel is filled with Neon (1 atm), at the downstream end a mosaic hexagonal mirrors focuses the Cherenkov light onto 2x1,000 photo detectors situated at the upstream end of the vessel (see Figure 12 and Figure 13). 


\subsection{The CHOD}

The charged hodoscope from NA48 [4] is re-used in NA62. This hodoscope is a system of scintillator counters with high granularity and good time resolution. It consist of 128 detection channels that are arranged in two layers. The read-out is based on the LAV FE and the TEL62 read-out and trigger board.

\subsection{The Muon Veto}

The muon veto system (MUV) consists of three sub-detectors, called MUV1, MUV2, and MUV3.

The first two modules (MUV1 and MUV2) follow directly the LKr calorimeter and work as hadronic calorimeters for measurement of deposited energies and shower shapes of incident particles. While MUV2 is the front module of the former NA48 hadron calorimeter (HAC), but turned by $180^{\circ}$, the MUV1 module is a newly constructed detector. Both modules are classic iron-scintillator sandwich calorimeters with 24 (MUV1) and 22 (MUV2) layers of scintillator strips. In both modules, the scintillator strips are oriented alternating in horizontal and vertical direction. In the MUV1 module light is collected by wave-length shifting (WLS) fibers, while the MUV2 module routes the scintillating light by light guides directly to photomultiplier tubes (see Figure 7).

The MUV3 detector is located after an additional Muon filter (80 $\mathrm{cm}$ of iron) serving as fast muon veto that can be used in the level 0 trigger. MUV3 is made of matrix of $5 \mathrm{~cm}$ thick scintillator tiles with direct photomultiplier read-out [2].

\subsection{Trigger DAQ}

The NA62 integrated Trigger and Data Acquisition system is based on a unified handling of the data completely in the digital domain to allow maximum offline control. A custom readout and trigger board, called TEL62, is used in most sub-detectors, this board is a further development from the TEL1 board that is used in LHCb [6], [7].

The trigger comprises a single hardware level (L0) based on CHOD, RICH, MUV, LKR and possibly the LAV, reducing the rate to $1 \mathrm{MHz}$ in $1 \mathrm{~ms}$ latency. At this rate, data is readout to a local PC farm where a software L1 trigger is performed and LKR data are read out. Sub-sequent event building and L2 triggering occurs in the same PC farm [8].

\section{Experience from the Technical Run}

The construction and installation of the NA62 experiments extends over 5 years and has, undeniably, a considerable complexity. The experiment plans a first physics run in autumn 2014. Due to the LHC shutdown (LS1) there will be no beam time for NA62 between December 2012 and summer 2014, it was therefore quite important to have a first run in autumn 2012. The goal for this Technical Run was not to set-up a complete 
experiment with a large number of read-out channels, but to test with a maximum number of sub-detectors the functionality of the apparatus, the read-out and the trigger.

Following that spirit most of the sub-systems (all except GTK and RICH) participated in the run with partly equipped detectors and with a quite limited amount of readout channels. A summary of the detector operation is given in Table 2 .

Table 2 Detector status during the Technical Run in autumn 2012

\begin{tabular}{|c|c|}
\hline Sub-System & Installation and Operation status during Technical Run \\
\hline KTAG & $\begin{array}{l}\text { KTAG installed. } 4 \text { out of } 8 \Phi \text {-sectors equipped to } 50 \% \text { with } \\
\text { photo- detectors and read-out. }\end{array}$ \\
\hline GTK & Was not installed for the Technical Run \\
\hline CHANTI & $\begin{array}{l}\text { Prototype detector (64 channels) used inside and outside vacuum } \\
\text { (positioned near LAV1) }\end{array}$ \\
\hline STRAW Tracker & $\begin{array}{l}\text { Chamber } 1 \text { installed equipped with } 2 \text { Modules: } \\
\mathrm{u} / \mathrm{v} \text { Module: Straws/Wires fully equipped } \\
\text { x/y Module: empty frame (no straws) made vacuum tight. } \\
\text { Readout limited to } 8 \mathrm{FE} \text { boards with } 2 \text { SRB prototype boards. } \\
\text { Standalone data stream }\end{array}$ \\
\hline RICH & Was not installed for the Technical Run \\
\hline CHOD / NHOD & $\begin{array}{l}\text { The NA48 CHOD and NHOD were reconditioned and equipped } \\
\text { with the LAV FE boards } \\
\text { Several small prototypes for future CHOD upgrade were installed } \\
\text { and tested. }\end{array}$ \\
\hline IRC & Same technology as SAC \\
\hline LKR & $\begin{array}{l}\text { NA48 Detector. SLM readout used ( } 30 \% \text { of the channels). Beta } \\
\text { version of cream prototype tested. }\end{array}$ \\
\hline MUV & MUV2 and MUV3 fully installed and operated \\
\hline SAC & Final detector installed. Temporary readout. \\
\hline $\begin{array}{l}\text { Vacuum tank and } \\
\text { vacuum system }\end{array}$ & $\begin{array}{l}85 \mathrm{~m} \text { (out of } 115 \mathrm{~m} \text { ) Vacuum tank and corresponding detector } \\
\text { elements were installed. } \\
\text { Primary pumping system and } 3 \text { (out of } 5 \text { ) cryo pumps were } \\
\text { installed. Vacuum pressure reached } 10^{-5} \mathrm{mbar}\end{array}$ \\
\hline
\end{tabular}

The lessons learnt from the Technical Run were multiple and certainly very important to complete the experiment:

- Most of the sub-detectors were brought for the first time into common operating conditions, which entailed operating the vacuum system with detectors installed and running as well as operating the detectors slow control systems remotely.

- The new beam line was tested and successfully tuned to good operating conditions.

- Time synchronisation of the different detectors was done. 
- The trigger and data acquisition systems were operated successfully, although at low rate. Two trigger schemes were tested and a list of open issues for returning trigger primitives from the sub-detectors to the L0 trigger processors was established.

- The data from all the detectors are used to compare their performance with the expectations.

- Last but not least it is possible to analyse definite event clusters, e.g. $\mathrm{K}^{+} \rightarrow \pi^{+} \pi^{0}$ allowing to $\mathrm{x}$-check detector performance with specific particles and constrained kinematics

Further results and conclusions from the Technical Run data analysis can be found in [9].

\section{References}

[1] Brod, J., Gorbahn, M., Stamou, E., Two-Loop Electroweak Corrections for the K -> pi nu antinu Decays Phys. Rev. D 83 (2011) 034030.

[2] NA62 Collaboration, Technical Design, NA62-10-07, http://cds.cern.ch/record/1404985.

[3] F. Anghinolfi et al. NINO: an ultra-fast and low-power front-end amplifier/discriminator ASIC designed for the multigap resistive plate chamber. Nucl. Instum. Methods A533 (2004) 183.

[4] NA48 Collaboration. The Beam and Detector for the NA48 Neutral Kaon CP Violation Experiment at CERN. Nucl. Instum. Methods A574 (2007) 433-471

[5] Ceccucci, A. et al. The NA62 Liquid Krypton calorimeter readout module J. Instrum. 6 (2011) C12017.

[6] Haefeli, G et al. he LHCb DAQ interface board TELL1. Nucl. Instrum. Methods , A560 (2006) 494-502

[7] Pedrischi E., Sozzi M., Spinella F. From TELL1 to TEL62. NA62-10-06 2010

[8] Avanzini,C. et al. The trigger and DAQ system for the NA62 experiment Nucl. Instrum. Methods A623 (2010) 543-545

[9] Ruggerio, G. et al. Status and Prospects for $\mathrm{K}+->$ pi+ nu nubar observation at CERN This conference, PoS (KAON2013) 032. 